\title{
ELEMENTARY DIFFERENCES BETWEEN THE ISOLS AND THE CO-SIMPLE ISOLS $\left(^{(}\right)$
}

\author{
BY \\ LOUISE HAY $\left(^{2}\right)$
}

1. Introduction. Let $E$ denote the nonnegative integers. For $\alpha, \beta \subseteq E$, $\alpha$ is recursively equivalent to $\beta$ if there is a 1-1 partial recursive function $p$ with $\alpha \subseteq$ domain $p$ and $p(\alpha)=\beta$; the equivalence class of $\alpha$ is denoted by $\langle\alpha\rangle$. A set $\alpha$ is isolated if it has no infinite recursively enumerable (r.e.) subset. The equivalence classes of isolated sets are called isols, and their collection is denoted by $\Lambda$. The elements of $\Lambda$ can be considered an "effective" analogue of the Dedekind finite cardinals; their properties were extensively studied by Dekker, Myhill, and Nerode (see, e.g., [2] and [6]). Isols $\langle\alpha\rangle$ of sets $\alpha$ such that $\alpha^{\prime}$ is r.e. are called co-simple isols, and their collection is denoted by $\Lambda_{z}$. The system $\Lambda_{z}$ was shown in [3] to exhibit much of the behavior of $\Lambda$; this presumably reflects the "effectiveness" common to the definitions of recursive equivalence and recursive enumerability, which makes it possible in many instances, given the existence of an isol with certain properties, to construct an r.e. set $\alpha$ such that $\left\langle\alpha^{\prime}\right\rangle$ has the required properties. The question of whether there exist elementary differences between $\Lambda$ and $\Lambda_{z}$ was left open in [3]. It is the purpose of this paper to exhibit differences in the first-order theories of addition and multiplication of $\Lambda$ and $\Lambda_{z}$.

More precisely, let $L$ denote a first-order functional calculus based on identity, addition, and multiplication, with individual variables $x_{1}, x_{2}, \ldots, x, y, z, \ldots$ and logical symbols $(\exists),(\forall), \wedge, \vee, \neg, \supset$. Given a system $(M,+, \cdot)$, a formula $B\left(x_{1}, \ldots, x_{n}\right)$ of $L$ whose only free variables are $x_{1}, \ldots, x_{n}$ and elements $X_{1}, \ldots, X_{n}$ of $M$, we say $B\left(X_{1}, \ldots, X_{n}\right)$ is true in $M$ if, when the quantified variables are interpreted as ranging over $M$, the result is a true statement in the theory of $(M,+, \cdot)$. The first-order theories of $(\Lambda,+, \cdot)$ and $\left(\Lambda_{z},+, \cdot\right)$ are both expressible in $L$, and we propose to exhibit a class of (closed) sentences $\left\{S_{\theta}\right\}$ of $L$ which are true in $\Lambda$ but false in $\Lambda_{z}$. We shall follow the usual practice of identifying $E$ with the finite elements of $\Lambda$ and $\Lambda_{z}$ and of thus considering the system $(E,+, \cdot)$ as a subsystem of $(\Lambda,+, \cdot)$ and $\left(\Lambda_{z},+, \cdot\right)$.

To define the sentences $S_{\theta}$ we shall require a formula of $L$ which defines $E$ in $\Lambda$ and in $\Lambda_{z}$, i.e., a formula with one free variable which, when interpreted in $\Lambda$ and $\Lambda_{z}$ respectively, is true of exactly the finite elements of those systems. Such a

Presented to the Society, April 5, 1966; received by the editors February 21, 1966.

(1) Research supported under National Science Foundation Grant GP-4537.

(2) The author is grateful to A. Nerode and A. B. Manaster for valuable discussions on the subject of this paper. 
formula is given for $\Lambda$ in [2], by the fact that the finite isols are exactly those comparable (under $\leqq$ ) to all other elements of $\Lambda$. It will be shown that this is also true in $\Lambda_{z}$, so that the same formula can be used to define $E$ in $\Lambda_{z}$. The proof of this fact will constitute Part 3 of this paper.

2. The sentences $S_{\theta}$. Let $P_{0}, P_{1}, \ldots$ be the sequence of positive primes. If $\theta: E \rightarrow E$ is any function and $X$ is an isol, we follow [1] in calling the sequence $\{\theta(n)\}$ the characteristic of $X$ if, for all $n$,

$$
P_{n}^{\theta(n)} \mid X \quad \text { and not } P_{n}^{\theta(n)+1} \mid X .
$$

It is proved in [1] that for each $\theta$, there is an isol $X$ having $\{\theta(n)\}$ as its characteristic. It will be shown that this fact is expressible by a sentence $S_{\theta}$ of $L$ when $\theta$ is arithmetically definable. It will also be shown that for a large class of such $\theta$, no $X \in \Lambda_{z}$ has $\{\theta(n)\}$ as its characteristic. Then for such $\theta, S_{\theta}$ will be true in $\Lambda$ but false in $\Lambda_{z}$.

THEOREM 1. Corresponding to each arithmetically definable function $\theta: E \rightarrow E$ whose representing predicate is not expressible in both 5-quantifier forms in the arithmetic hierarchy, there is a sentence $S_{\theta}$ of $L$ which is true in $(\Lambda,+, \cdot)$ but false in $\left(\Lambda_{z},+, \cdot\right)$.

Proof. We shall henceforth assume that a formula of $L$ written $B\left(x_{1}, \ldots, x_{m}\right)$ contains no free variables other than $x_{1}, \ldots, x_{m}$. Let $\theta: E \rightarrow E$ be arithmetically definable, and let $R_{\theta}(m, n)$ be its representing predicate. There is thus a prenex formula $B_{\theta}(y, z)$ of $L$ such that, for all $m, n \in E$,

$$
m=\theta(n) \leftrightarrow R_{\theta}(m, n) \leftrightarrow B_{\theta}(m, n) \text { is true in } E .
$$

Let $h: E \times E \rightarrow E$ be the recursive function with representing predicate $S(k, m, n)$ defined by

$$
S(k, m, n) \leftrightarrow k=h(m, n) \leftrightarrow k=P_{n}^{m} .
$$

By the arithmetic definability of recursive relations, there is a prenex formula $C(x, y, z)$ of $L$ such that for all $k, m, n \in E$,

$$
S(k, m, n) \leftrightarrow C(k, m, n) \text { is true in } E .
$$

(D3) Let Fin $(x)$ denote the following formula of $L$ :

$$
(\forall y)(\exists z)(x+z=y \vee y+z=x) .
$$

A conjunction $\operatorname{Fin}\left(x_{1}\right) \wedge \cdots \wedge \operatorname{Fin}\left(x_{m}\right)$ will be abbreviated by $\operatorname{Fin}\left(x_{1}, \ldots, x_{m}\right)$.

(D4) For any prenex formula $B\left(x_{1}, \ldots, x_{m}\right)$ of $L$, define a corresponding formula $B^{E}\left(x_{1}, \ldots, x_{m}\right)$ of $L$ by induction on the number of quantifiers, as follows:

(a) If $B\left(x_{1}, \ldots, x_{m}\right)$ has no quantifiers, let

$$
B^{E}\left(x_{1}, \ldots, x_{m}\right)=B\left(x_{1}, \ldots, x_{m}\right) .
$$

(b) If $B\left(x_{1}, \ldots, x_{m}\right)$ is $(\forall y) C\left(x_{1}, \ldots, x_{m}, y\right)$ and $C^{E}\left(x_{1}, \ldots, x_{m}, y\right)$ has been defined, let

$$
B^{E}\left(x_{1}, \ldots, x_{m}\right)=(\forall y)\left(\operatorname{Fin}(y) \supset C^{E}\left(x_{1}, \ldots, x_{m}, y\right)\right) .
$$


(c) If $B\left(x_{1}, \ldots, x_{m}\right)$ is $(\exists y) C\left(x_{1}, \ldots, x_{m}, y\right)$ and $C^{E}\left(x_{1}, \ldots, x_{m}, y\right)$ has been defined, let

$$
B^{E}\left(x_{1}, \ldots, x_{m}\right)=(\exists y)\left(\operatorname{Fin}(y) \wedge C^{E}\left(x_{1}, \ldots, x_{m}, y\right)\right) .
$$

The definition of $B^{E}\left(x_{1}, \ldots, x_{m}\right)$ evidently serves to restrict the range of the quantifiers in $B\left(x_{1}, \ldots, x_{m}\right)$ to $\{y \mid \operatorname{Fin}(y)\}$.

In terms of the formulas introduced in (D1)-(D4), let $A_{\theta}(x)$ be the following formula of $L$ :

$(\forall y)(\forall z)(\forall u)(\forall v)\left[\left(\operatorname{Fin}(y, z, u, v) \wedge B_{\theta}^{E}(y, z) \wedge C^{E}(u, y, z) \wedge C^{E}(v, y+1, z)\right)\right.$

$$
\supset((\exists t)(x=t u) \wedge \neg(\exists t)(x=t v))] .
$$

Finally, let $S_{\theta}$ be the sentence $(\exists x) A_{\theta}(x)$. It remains to show that:

(1) $S_{\theta}$ is true in $\Lambda$.

(2) $S_{\theta}$ is true in $\Lambda_{z}$ only if $R_{\theta}(m, n)$ is expressible in both 5-quantifier forms.

Lemma 1.1. Let $X \in \Lambda$. Then $\operatorname{Fin}(X)$ is true in $\Lambda$ if and only if $X \in E$.

Proof. This is shown in [2, p. 103].

LEMMA 1.2. Let $B\left(x_{1}, \ldots, x_{m}\right)$ be a prenex formula of $L$, and let $X_{1}, \ldots, X_{m} \in E$. Then $B\left(X_{1}, \ldots, X_{m}\right)$ is true in $E$ if and only if $B^{E}\left(X_{1}, \ldots, X_{m}\right)$ is true in $\Lambda$.

Proof. By induction on the number of quantifiers in $B\left(x_{1}, \ldots, x_{m}\right)$ :

(a) If $B\left(x_{1}, \ldots, x_{m}\right)$ has no quantifiers, then since $(E,+, \cdot)$ is a subsystem of $(\Lambda,+, \cdot)$, the following are equivalent:

(a1) $B\left(X_{1}, \ldots, X_{m}\right)$ is true in $E$,

(a2) $B\left(X_{1}, \ldots, X_{m}\right)$ is true in $\Lambda$,

(a3) $B^{E}\left(X_{1}, \ldots, X_{m}\right)$ is true in $\Lambda$.

(b) Assume that $B\left(x_{1}, \ldots, x_{m}\right)$ is $(\forall y) C\left(x_{1}, \ldots, x_{m}, y\right)$ and that the lemma holds for $C\left(x_{1}, \ldots, x_{m}, y\right)$. Then by Lemma 1.1, the following are equivalent:

(b1) $B\left(X_{1}, \ldots, X_{m}\right)$ is true in $E$,

(b2) for all $Y \in E, C\left(X_{1}, \ldots, X_{m}, Y\right)$ is true in $E$,

(b3) for all $Y \in E, C^{E}\left(X_{1}, \ldots, X_{m}, Y\right)$ is true in $\Lambda$,

(b4) $(\forall y)\left(\operatorname{Fin}(y) \supset C^{E}\left(X_{1}, \ldots, X_{m}, y\right)\right)$ is true in $\Lambda$,

(b5) $B^{E}\left(X_{1}, \ldots, X_{m}\right)$ is true in $\Lambda$.

(c) Similarly if $B\left(x_{1}, \ldots, x_{m}\right)$ is $(\exists y) C\left(x_{1}, \ldots, x_{m}, y\right)$.

Lemma 1.3. Let $X \in \Lambda$. Then $\{\theta(n)\}$ is the characteristic of $X$ if and only if $A_{\theta}(X)$ is true in $\Lambda$.

Proof. By (D1) and (D2), Lemma 1.2, and Lemma 1.1, the following are equivalent:

(1) $\{\theta(n)\}$ is the characteristic of $X$,

(2) for all $Z \in E, Y=\theta(Z)$ and $U=P_{Z}^{Y}$ and $V=P_{Z}^{Y+1}$ together imply

$$
[(\exists t)(X=t U) \wedge \neg(\exists t)(X=t V)]
$$

is true in $\Lambda$, 
(3) for all $Y, Z, U, V \in E,\left[B_{\theta}(Y, Z) \wedge C(U, Y, Z) \wedge C(V, Y+1, Z)\right]$ true in $E$ implies $[(\exists t)(X=t U) \wedge \neg(\exists t)(X=t V)]$ true in $\Lambda$,

(4) for all $Y, Z, U, V \in E,\left[B_{\theta}^{E}(Y, Z) \wedge C^{E}(U, Y, Z) \wedge C^{E}(V, Y+1, Z)\right]$ true in $\Lambda$ implies $[(\exists t)(X=t U) \wedge \neg(\exists t)(X=t V)]$ true in $\Lambda$,

(5) for all $Y, Z, U, V \in \Lambda$,

$$
\left[\operatorname{Fin}(Y, Z, U, V) \wedge B_{\theta}^{E}(Y, Z) \wedge C^{E}(U, Y, Z) \wedge C^{E}(V, Y+1, Z)\right]
$$

true in $\Lambda$ implies $[(\exists t)(X=t U) \wedge \neg(\exists t)(X=t V)]$ true in $\Lambda$,

(6) $A_{\theta}(X)$ is true in $\Lambda$.

Lemma 1.4. (a) If $X \in \Lambda_{z}, Y \in \Lambda$ and $(\exists t)(Y+t=X)$ is true in $\Lambda$ then $Y \in \Lambda_{z}$.

(b) If $X \in \Lambda_{z}$ and $U \in E$, then $(\exists t)(X=t U)$ is true in $\Lambda$ if and only if it is true in $\Lambda_{z}$.

Proof. Part (a) is Theorem 56(b) of [2]. For (b), assume that $X \in \Lambda_{z}$ and $U \in E$. One direction is trivial. Now assume that for some $T \in \Lambda, X=T U$. If $U=0$, then $X=0$. If $U>0$, then $X=T+(U-1) T$, so that by (a), $T \in \Lambda_{z}$. In either case, $(\exists t)(X=t U)$ is true in $\Lambda_{z}$.

Lemma 1.5. Let $X \in \Lambda_{z}$. Then $\operatorname{Fin}(X)$ is true in $\Lambda_{z}$ if and only if $X \in E$.

Proof. Assume $X \in E$ and $Y \in \Lambda_{z}$. We require some $Z \in \Lambda_{z}$ such that $X+Z=Y$ or $Y+Z=X$. Now by Lemma 1.1 , there is a $Z \in \Lambda$ such that $X+Z=Y$ or $Y+Z=X$. In the first case $Z \in \Lambda_{z}$ by Lemma 1.4(a); in the second case, $X \in E$ and $Z \leqq X$ implies $Z \in E \subseteq \Lambda_{z}$. Thus in either case, $Z \in \Lambda_{z}$ and Fin $(X)$ is true in $\Lambda_{z}$.

The converse will follow from Theorem 2 of Part 3, in which it is proved that $X \in \Lambda_{z}-E$ implies $\operatorname{Fin}(X)$ is false in $\Lambda_{z}$.

LEMMA 1.6. Let $B\left(x_{1}, \ldots, x_{m}\right)$ be a prenex formula of $L$, and let $X_{1}, \ldots, X_{m} \in E$. Then $B\left(X_{1}, \ldots, X_{m}\right)$ is true in $E$ if and only if $B^{E}\left(X_{1}, \ldots, X_{m}\right)$ is true in $\Lambda_{z}$.

Proof. Exactly like the proof of Lemma 1.2, replacing use of Lemma 1.1 by use of Lemma 1.5.

Lemma 1.7. Let $X \in \Lambda_{z}$. Then $\{\theta(n)\}$ is the characteristic of $X$ if and only if $A_{\theta}(X)$ is true in $\Lambda_{z}$.

Proof. By Lemma 1.4(b), (D1), and (D2), Lemma 1.6, and Lemma 1.5, the following are equivalent:

(1) $\{\theta(n)\}$ is the characteristic of $X$,

(2) for all $Z \in E, Y=\theta(Z)$ and $U=P_{Z}^{Y}$ and $V=P_{Z}^{Y+1}$ together imply

$$
[(\exists t)(X=t U) \wedge \neg(\exists t)(X=t V)]
$$

is true in $\Lambda$,

(3) for all $Z \in E, Y=\theta(Z)$ and $U=P_{Z}^{Y}$ and $V=P_{Z}^{Y+1}$ together imply

$$
[(\exists t)(X=t U) \wedge \neg(\exists t)(X=t V)]
$$

is true in $\Lambda_{z}$, 
(4) for all $Y, Z, U, V \in E,\left[B_{\theta}^{E}(Y, Z) \wedge C^{E}(U, Y, Z) \wedge C^{E}(V, Y+1, Z)\right]$ true in $\Lambda_{z}$ implies $[(\exists t)(X=t U) \wedge \neg(\exists t)(X=t V)]$ true in $\Lambda_{z}$,

(5) $A_{\theta}(X)$ is true in $\Lambda_{z}$.

We now introduce some recursion-theoretic notation. This will be largely informal, and such formalism as we use derives from [4]. The notation $(\quad),(E)$, $\&, \vee, \neg, \rightarrow$ of a first-order predicate calculus will be used for notational convenience, and is not to be confused with the formal symbolism of $L$. Let $q_{0}, q_{1}, \ldots$ be a Kleene enumeration of all partial recursive functions of one variable. If $w_{k}=$ range $q_{k}$, then $w_{0}, w_{1}, \ldots$ is an enumeration of all r.e. sets and if $X \in \Lambda_{z}$, then $X=\left\langle w_{e}^{\prime}\right\rangle$ for some $e$; we call $e$ an index of $X$. Let $p_{0}, p_{1}, \ldots$ be an effective enumeration of all 1-1 partial recursive functions, given by a recursive function $g$ such that $p_{k} \simeq q_{g(k)}$ for each $k$. We note that

$$
\begin{aligned}
z=p_{k}(x) \leftrightarrow & z=q_{g(k)}(x) \leftrightarrow(E y)\left(T_{1}^{1}(g(k), x, y) \& z=U(y)\right), \\
\langle\alpha\rangle=\langle\beta\rangle \leftrightarrow & (E k)\left(\alpha \subseteq \operatorname{domain} p_{k} \& p_{k}(\alpha)=\beta\right) \\
\leftrightarrow & {\left[(x)\left(x \in \alpha^{\prime} \vee(E z)\left(z=p_{k}(x) \& z \in \beta\right)\right)\right.} \\
& \left.\&(z)\left(z \in \beta^{\prime} \vee(E x)\left(x \in \alpha \& z=p_{k}(x)\right)\right)\right]
\end{aligned}
$$

LEMMA 1.8. Let $Q(a, b, m, n)$ denote the number-theoretic predicate:

$$
\left\langle w_{a}^{\prime}\right\rangle=P_{n}^{m} \cdot\left\langle w_{b}^{\prime}\right\rangle \text {. }
$$

Then $Q(a, b, m, n)$ is expressible in form $E A E A$ in the arithmetic hierarchy.

Proof. We show that a defining expression for $Q(a, b, m, n)$ in terms of quantifiers and recursive predicates can be brought to $E A E A$ prenex form by means of the Tarski-Kuratowski algorithm described in [7]. Let $h$ be the recursive function defined by $h(m, n)=P_{m}^{n}$ and, for $i \in E$, let

$$
\begin{aligned}
\beta_{i} & =\left\{2^{i} 3^{x} \mid x \in w_{b}^{\prime}\right\}, \\
\beta_{b, m, n} & =\beta_{0} \cup \cdots \cup \beta_{h(m, n)-1} .
\end{aligned}
$$

Then $\left\langle\beta_{i}\right\rangle=\left\langle w_{b}^{\prime}\right\rangle$ for each $i$, so that $\left\langle\beta_{b, m, n}\right\rangle=P_{m}^{n} \cdot\left\langle w_{b}^{\prime}\right\rangle$ and

$$
\begin{aligned}
& Q(a, b, m, n) \leftrightarrow\left\langle w_{a}^{\prime}\right\rangle=P_{m}^{n} \cdot\left\langle w_{b}^{\prime}\right\rangle=\left\langle\beta_{b, m, n}\right\rangle \\
& \leftrightarrow(E k)\left[(x)\left(x \in w_{a} \vee(E z)\left(z=p_{k}(x) \& z \in \beta_{b, m, n}\right)\right)\right. \\
&\left.\quad \&(z)\left(z \in \beta_{b, m, n}^{\prime} \vee(E x)\left(x \in w_{a}^{\prime} \& z=p_{k}(x)\right)\right)\right] .
\end{aligned}
$$

by (A2). Now $x \in w_{a}$ has $E$ form, since $w_{a}$ is r.e., $z=p_{k}(x)$ has $E$ form, by (A1), and $z \in \beta_{b, m, n} \leftrightarrow(E u)(E v)\left(v \in w_{b}^{\prime} \& u<h(m, n) \& z=2^{u} 3^{v}\right)$, which can be brought to form $E A$. Thus $Q(a, b, m, n)$ has form

$$
E[A(E \vee E(E \& E A)) \& A(A E \vee E(A \& E))]
$$

which by the algorithm can be reduced, in sequence, to $E[A(E \vee E A) \& A(A E \vee E A)], \quad E[A E A \&(A E \vee A E A)], \quad E A E A$. 
Lemma 1.9. Let $X \in \Lambda_{z}$, and assume that $\{\theta(n)\}$ is the characteristic of $X$. Then the representing predicate of $\theta$ is expressible in both 5-quantifier forms in the arithmetic hierarchy.

Proof. Let $X=\left\langle w_{a}^{\prime}\right\rangle$ have characteristic $\{\theta(n)\}$, and let $R_{\theta}(m, n)$ be the representing predicate of $\theta$, i.e.,

$$
R_{\theta}(m, n) \leftrightarrow m=\theta(n) \quad \text { for all } m, n \in E .
$$

Then, since the characteristic of $X$ is uniquely determined,

$$
\begin{aligned}
R_{\theta}(m, n) & \leftrightarrow P_{n}^{m}\left|X \& \neg P_{n}^{m+1}\right| X \\
& \leftrightarrow(E Y)_{Y \in \Lambda}\left(X=P_{n}^{m} Y\right) \& \neg(E Y)_{Y \in \Lambda}\left(X=P_{n}^{m+1} Y\right) \\
& \leftrightarrow(E Y)_{Y \in \Lambda_{z}}\left(X=P_{n}^{m} Y\right) \& \neg(E Y)_{Y \in \Lambda_{z}}\left(X=P_{n}^{m+1} Y\right),
\end{aligned}
$$

by Lemma 1.4(b)

$$
\begin{aligned}
& \leftrightarrow(E b)\left(\left\langle w_{a}^{\prime}\right\rangle=P_{n}^{m}\left\langle w_{b}^{\prime}\right\rangle\right) \& \neg(E b)\left(\left\langle w_{a}^{\prime}\right\rangle=P_{n}^{m+1}\left\langle w_{b}^{\prime}\right\rangle\right) \\
& \leftrightarrow(E b) Q(a, b, m, n) \&(b) \neg Q(a, b, m+1, n),
\end{aligned}
$$

which by Lemma 1.8 has form $E(E A E A) \& A(A E A E)$ which reduces to $E A E A \& A E A E$. As is well known, this form is recursive in the 4-quantifier form of highest degree, or, equivalently, can be written in both 5-quantifier forms.

Proof of Theorem 1. Let $\theta$ be any arithmetically definable function whose representing predicate $R_{\theta}(m, n)$ is not expressible in both 5-quantifier forms. Then:

(1) By [1, Theorem T1] there is an $X \in \Lambda$ which has $\{\theta(n)\}$ as its characteristic. By Lemma 1.3, this implies $A_{\theta}(X)$ is true in $\Lambda$, so that $S_{\theta}$ is true in $\Lambda$.

(2) Assume $S_{\theta}$ is true in $\Lambda_{z}$; then for some $X \in \Lambda_{z}, A_{\theta}(X)$ is true in $\Lambda_{z}$. By Lemma 1.7, this implies $\{\theta(n)\}$ is the characteristic of $X$, from which it follows by Lemma 1.9 that $R_{\theta}(m, n)$ is expressible in both 5-quantifier forms. Since this is a contradiction, we conclude that $S_{\theta}$ is false in $\Lambda_{z}$.

REMARK. The sentences $S_{\theta}$ chosen for Theorem 1 are merely illustrative of a type of sentence which can serve to distinguish between the first-order theories of $(\Lambda,+, \cdot)$ and $\left(\Lambda_{z},+, \cdot\right)$. Theorem $\mathrm{T} 1$ of [1], which was applied above, is a special case of the "extended Chinese remainder theorem for isols" [5, Theorem 4.5]. Other instances of the latter could be similarly used to yield elementary differences between $\Lambda$ and $\Lambda_{z}$.

3. First-order characterization of $E$ in $\Lambda_{z}$. It remains to show that the finite isols are the only elements of $\Lambda_{z}$ comparable to all other elements of $\Lambda_{z}$. This requires the direct construction of r.e. sets, for which the natural tool is the "priority" method in its various manifestations [8]. A scheme for adapting this method to the construction of co-simple isols was described in [3]; unfortunately it does not appear to be sufficiently general to handle the present case. 
Theorem 2. Assume $X \in \Lambda_{z}-E$. Then there is a $Y \in \Lambda_{z}$ such that $X \$ Y$ and $Y \$ X$.

Proof. The recursion-theoretic notation is that introduced for the proof of Lemma 1.8. We assume an effective procedure for simultaneously generating all r.e. sets, and denote by $w_{k}^{u}$ the finite set of elements of $w_{k}$ generated at stages $t \leqq u$. Let $R_{0}, R_{1}, \ldots$ be a partition of $E$ into infinite disjoint recursive sets. We adopt the following notation for purposes of abbreviation:

$$
\alpha \prec_{k} \beta \equiv \alpha \subseteq \text { domain } p_{k} \text { and } p_{k}(\alpha) \subseteq \beta \text {. }
$$

Thus $\langle\alpha\rangle \leqq\langle\beta\rangle$ only if $(E k)\left(\alpha \prec{ }_{k} \beta\right)$.

$$
\begin{aligned}
& \pi_{k}^{t}(x)=1+U\left((\mu y)_{y \leqq t} T_{1}^{1}(g(k), x, y)\right), \quad \text { if }(E y)_{y \leqq t} T_{1}^{1}(g(k), x, y), \\
& =0 \text { otherwise, } \\
& \qquad \begin{aligned}
p_{k}^{t}(x) & =\pi_{k}^{t}(x)-1, \quad \text { if } \pi_{k}^{t}(x)>0 \\
& =0 \text { otherwise. }
\end{aligned}
\end{aligned}
$$

$p_{k}^{t}$ is thus a recursive function whose value corresponds roughly to the result of performing $t$ steps in the computation of $p_{k}(x)$. It is evident that $\pi_{k}^{t}(x)$ and $p_{k}^{t}(x)$ are bounded, nondecreasing functions of $t$ and that

$$
\begin{aligned}
\lim _{t} p_{k}^{t}(x) & =p_{k}(x), \quad \text { if } x \in \text { domain } p_{k}, \\
& =0 \text { otherwise. }
\end{aligned}
$$

Now assume $X \in \Lambda_{z}-E$, so that $X=\left\langle w_{a}^{\prime}\right\rangle$ where $w_{a}^{\prime}$ is immune. Instructions will be given for generating an r.e. set $\gamma$ which will satisfy the following "requirements," for each $k$ :

$\left(1_{k}\right) w_{k}$ infinite $\rightarrow w_{k} \cap \gamma \neq \varnothing$,

$\left(2_{k}\right)$ not $\gamma^{\prime} \prec_{k} w_{a}^{\prime}$,

$\left(3_{k}\right)$ not $w_{a}^{\prime} \prec_{k} \gamma^{\prime}$.

This will evidently give $Y=\left\langle\gamma^{\prime}\right\rangle \in \Lambda_{z}$, with $Y \$ X$ and $X \$ Y$. We will define by simultaneous induction on $t$, recursive functions $F(k, t), G(k, t)$, and $H(k, t)$, and will generate $\gamma$ by putting into it at each stage $t$ the values $F(k, t), G(k, t), H(k, t)$ for $k \leqq t$. The gist of the construction is as follows: To satisfy requirement $\left(2_{k}\right)$, we try to keep in $\gamma^{\prime}$ some $x$ for which $p_{k}(x) \notin w_{a}^{\prime}$; to satisfy requirement $\left(1_{k}\right)$, we try to put into $\gamma$ an element of $w_{k}$; and to satisfy requirement $\left(3_{k}\right)$, we try to put into $\gamma$ the number $p_{k}(z)$ for some $z \in w_{a}^{\prime}$. These are evidently conflicting requirements, and we resolve this conflict by the following device:

At stage $t$, we "tag" the least $x \in R_{k}-\gamma^{t-1}$ for which it appears that $p_{k}(x) \notin w_{a}^{\prime}$, and put into $\gamma, G(k, t)=$ the next larger element of $R_{k}-\gamma^{t-1}$. The fact that $w_{a}^{\prime}$ is immune and that hence there is no infinite r.e. sequence $\left\{x_{i}\right\}$ such that $p_{k}\left(x_{i}\right) \in w_{a}^{\prime}$ for all $i$, will insure that (i) only finitely many elements of $R_{k}$ are ever "tagged," 
(ii) for some "tagged" $x \in R_{k}, p_{k}(x) \notin w_{a}^{\prime}$ and $x$ is never put in $\gamma$, and (iii) $R_{k} \cap \gamma^{\prime}$ is finite.

Since the "naive" attempts at satisfying requirements $\left(1_{k}\right)$ and $\left(3_{k}\right)$ described above would interfere with the termination of the "tagging" process, we modify them slightly as follows: The value of $F(k, t)$ represents an attempt to put into $\gamma$ an element of $w_{k}-\bigcup_{j \leqq k} R_{j}$, and that of $H(k, t)$ an attempt to put into $\gamma, p_{k}(z)$ for some $z \in w_{a}^{\prime}$ for which $p_{k}(z) \notin \bigcup_{j<k} R_{j}$. These attempts will fail only if (1) $w_{k} \subseteq \bigcup_{j \leqq k} R_{j}$ or (2) $p_{k}\left(w_{a}^{\prime}\right) \subseteq \bigcup_{j<k} R_{j}$. In the first case, $w_{k} \cap \gamma^{\prime} \subseteq\left(\bigcup_{j \leqq k} R_{j}\right) \cap \gamma^{\prime}$ which is finite, so that either $w_{k}$ is finite or $w_{k} \cap \gamma \neq \varnothing$; in the second case, $p_{k}\left(w_{a}^{\prime}\right) \cap \gamma^{\prime} \subseteq\left(\bigcup_{j<k} R_{j}\right) \cap \gamma^{\prime}$ which is finite, so that either $p_{k}(z)$ is undefined for some $z \in w_{a}^{\prime}$ or $p_{k}(z) \in \gamma$ for some $z \in w_{a}^{\prime}$. Thus in either case, the relevant requirement is satisfied anyway.

We proceed to the formal construction and the proof (by induction on $k$ ) that all requirements are eventually satisfied. Define $F(k, t), G(k, t), H(k, t)$ and auxiliary recursive functions $n(k, t), r(k, t), x(i, k, t), z(i, t), v(k, t), s(k, t)$ as follows:

Stage 0. For all $k$ and $i$, let $F(k, 0)=G(k, 0)=H(k, 0)=n(k, 0)=r(k, 0)$ $=x(i, k, 0)=z(i, 0)=v(k, 0)=s(k, 0)=0$. Let $\gamma^{0}=\{0\}$.

Stage $t>0$. For $k>t$ and all $i$, let $F(k, t)=G(k, t)=H(k, t)=n(k, t)=r(k, t)$ $=x(i, k, t)=v(k, t)=s(k, t)=0$.

(a) For $0 \leqq k \leqq t$, let

$$
v=v(k, t)=(\mu v)_{v \leqq t}\left(\left(w_{k}^{t}-\bigcup_{j \leqq k} R_{j}=\varnothing \& v=0\right) \vee w_{k}^{v}-\bigcup_{j \leqq k} R_{j} \neq \varnothing\right),
$$

$F(k, t)=(\mu x)\left(\left(w_{k}^{v}-\bigcup_{j \leqq k} R_{j}=\varnothing \quad \& \quad x=0\right) \vee x \in w_{k}^{v}-\bigcup_{j \leqq k} R_{j}\right)$.

(b) For $0 \leqq k \leqq t$, let

$$
\begin{aligned}
& x(0, k, t)=(\mu x)\left(x \in R_{k}-\gamma^{t-1}\right), \\
& x(i+1, k, t)=(\mu x)\left(x>x(i, k, t) \& x \in R_{k}-\gamma^{t-1}\right) \quad i=0,1, \ldots, \\
& n=n(k, t)=(\mu i)_{i \leqq t}\left(\pi_{k}^{t} x(i, k, t)=0 \vee\left(\pi_{k}^{t} x(i, k, t)>0\right.\right. \\
& \left.\left.\& p_{k}^{t} x(i, k, t) \in w_{a}^{t}\right) \vee i=t\right), \\
& G(k, t)=x(n+1, k, t) \quad \text { if } \pi_{k}^{t} x(n, k, t)=0 \vee\left(\pi_{k}^{t} x(n, k, t)>0\right. \\
& \left.\& p_{k}^{t} x(n, k, t) \in w_{a}\right) \\
& =0 \text { otherwise. }
\end{aligned}
$$

(c) Let

$$
\begin{aligned}
z(0, t) & =(\mu z)\left(z \notin w_{a}^{t}\right), \\
z(i+1, t) & =(\mu z)\left(z>z(i, t) \& z \notin w_{a}^{t}\right) \quad i=0,1, \ldots, \\
H(0, t) & =p_{k}^{t} z(0, t)
\end{aligned}
$$


For $0<k \leqq t$, let

$$
\begin{aligned}
& r=r(k, t)=1+\sum_{j<k}(1+n(j, t)), \\
& s=s(k, t)=(\mu i)_{i \leqq r}\left(\pi_{k}^{t} z(i, t)=0 \vee\left(\pi_{k}^{t} z(i, t)>0 \& p_{k}^{t} z(i, t) \in \bigcap_{j k} R_{j}^{\prime}\right) \vee i=r\right), \\
& \begin{aligned}
H(k, t) & =p_{k}^{t} z(s, t) \text { if } \pi_{k}^{t} z(s, t)>0 \& p_{k}^{t} z(s, t) \in \bigcap_{j<k} R_{j}^{\prime}, \\
& =0 \text { otherwise. }
\end{aligned}
\end{aligned}
$$

Finally, let $\gamma^{t}=\gamma^{t-1} \cup\left(\bigcup_{k \leqq t}\{F(k, t), G(k, t), H(k, t)\}\right)$.

REMARK. The following easily verified facts are noted here for future reference:

(R1) $z(i, t)$ and $x(i, k, t)$ are strictly increasing functions of $i$.

(R2) $F(j, t) \neq 0 \rightarrow F(j, t) \in \bigcap_{k<j} R_{k}^{\prime}$.

(R3) $H(j, t) \neq 0 \rightarrow H(j, t) \in \bigcap_{k<j} R_{k}^{\prime}$.

(R4) $(G(j, t) \neq 0 \& n=n(j, t)) \rightarrow\left(\pi_{k}^{t} x(n, j, t)=0 \vee p_{k}^{t} x(n, j, t) \in w_{a}^{t}\right)$.

(R5) $G(j, t) \neq 0 \rightarrow G(j, t) \in R_{j}$.

LEMMA 2.1. For each $k$,

(a) $\lim _{t} F(k, t)$ exists,

(b) if $w_{k}-\bigcup_{j \leqq k} R_{j} \neq \varnothing$, then $w_{k} \cap \gamma \neq \varnothing$.

Proof. Case 1. $w_{k}-\bigcup_{j \leqq k} R_{j}=\varnothing$.

Then for all $t, w_{k}^{t}-\bigcup_{j \leqq k} R_{f}=\varnothing$, so that $v(k, t)=F(k, t)=0$. This proves (a), and (b) holds trivially.

Case 2. Otherwise. Let

$$
\begin{aligned}
& t^{*}=(\mu t)\left(t \geqq k \& w_{k}^{t}-\bigcup_{j \leqq k} R_{j} \neq \varnothing\right), \\
& x^{*}=(\mu x)\left(x \in w_{k}^{t^{*}}-\bigcup_{j \geqq k} R_{j}\right) .
\end{aligned}
$$

Then for all $t \geqq t^{*}, v(k, t)=t^{*}$ and $F(k, t)=x^{*}$, which proves (a). Part (b) follows since $x^{*} \in w_{k}$ and $x^{*}=F\left(k, t^{*}\right) \in \gamma^{t^{*}} \subseteq \gamma$.

LEMMA 2.2. (a) $\lim _{t} H(0, t)$ exists,

(b) not $w_{a}^{\prime} \prec_{0} \gamma^{\prime}$.

Proof. Let $z^{*}=(\mu z)\left(z \in w_{a}^{\prime}\right)$, so that $(z)_{z<z^{*}}(E t)\left(z \in w_{a}^{t}\right)$. Let

$$
t^{*}=(\mu t)(z)_{z<z^{*}}\left(z \in w_{a}^{t}\right) \text {. }
$$

Then for all $t \geqq t^{*}, z(0, t)=z^{*}$.

Case 1. $z^{*} \notin$ domain $p_{0}$. Then $\pi_{0}^{t}\left(z^{*}\right)=0$ for all $t$, so that for all $t \geqq t^{*}, H(0, t)$ $=\pi_{0}^{t}\left(z^{*}\right)=0$, which proves (a). Part (b) holds since $z^{*} \in w_{a}^{\prime}$ implies $w_{a}^{\prime} \nsubseteq$ domain $p_{0}$. Case 2. Otherwise. Then for some $u, \pi_{0}^{u}\left(z^{*}\right)>0$ and $p_{0}^{u}\left(z^{*}\right)=p_{0}\left(z^{*}\right)$. Let $u^{*}$ $=\max \left(t^{*}, u\right)$. Then for all $t \geqq u^{*}, H(0, t)=p_{0}^{t}\left(z^{*}\right)=p_{0}\left(z^{*}\right)$, which proves (a). Part (b) holds since $z^{*} \in w_{a}^{\prime}$ while $p_{0}\left(z^{*}\right)=H\left(0, u^{*}\right) \in \gamma^{u^{*}} \subseteq \gamma$.

In the following, $c(\alpha)$ denotes cardinality $\alpha$. 
LeMma 2.3. If $\lim _{t} H(j, t)$ exists for all $j \leqq k$, then

(a) $\lim _{t} n(k, t)$ exists,

(b) $c\left(R_{k} \cap \gamma^{\prime}\right)=1+\lim _{t} n(k, t)$,

(c) not $\gamma^{\prime} \prec_{k} w_{a}^{\prime}$.

Proof. This will be divided into steps (2.3.1)-(2.3.5), each of which has as hypothesis

$$
\lim _{t} H(j, t) \text { exists for all } j \leqq k .
$$

(2.3.1) There is a stage $u>k$ such that

$$
(x)\left[\left(x \in R_{k} \&(E t)_{t>u}(E j)(x=F(j, t) \vee x=H(j, t))\right) \rightarrow x \in \gamma^{u}\right]
$$

(i.e., after stage $u$ no new elements of $R_{k}$ are added to $\gamma$ to satisfy requirements $\left(1_{j}\right)$ or $\left(3_{j}\right)$ for any $j$.)

Proof. Let $u$ be chosen so large that for all $j \leqq k$ and all $t \geqq u$,

$$
\begin{gathered}
H(j, t)=\lim _{t} H(j, t)=H_{j} \quad \text { (which exists by the hypothesis), } \\
F(j, t)=\lim _{t} F(j, t)=F_{j} \quad \text { (which exists by Lemma 2.1). }
\end{gathered}
$$

If $x=0$, the conclusion holds trivially since $x \in \gamma^{u}$ for all $u$. If $x \neq 0, x \in R_{k}$ and $(x=F(j, t) \vee x=H(j, t))$ then by (R2) and (R3) it follows that $k \geqq j$, while by choice of $u, t>u$ implies

$$
x=F(j, t)=F_{j}=F(j, u) \vee x=H(j, t)=H_{j}=H(j, u) .
$$

This, together with $j \leqq k<u$, implies $x \in \gamma^{u}$.

Let $u_{k}=$ the least $u$ satisfying (2.3.1). We define by simultaneous induction on $t$ two partial recursive functions $t_{k}(i)$ and $f_{k}(i)$, as follows:

$$
t_{k}(0)=u_{k}+1, \quad f_{k}(0)=(\mu x)\left(x \in R_{k}-\gamma^{u_{k}}\right) .
$$

Now assume that for all $j<i, t_{k}(j)$ and $f_{k}(j)$ have been defined.

Case 1. $(E t)\left(t>t_{k}(i-1) \&(j)_{j<i}\left(\pi_{k}^{t} f_{k}(j)>0 \& p_{k}^{t} f_{k}(j) \notin w_{a}^{t}\right)\right)$. Then define

$$
\begin{aligned}
t_{k}(i) & =\text { the least such } t, \\
f_{k}(i) & =(\mu x)\left(x>f_{k}(i-1) \& x \in R_{k}-\gamma^{t_{k}(i)-1}\right) .
\end{aligned}
$$

Case 2. Otherwise. Then $t_{k}(i)$ and $f_{k}(i)$ are undefined. It is evident that $t_{k}$ and $f_{k}$ are strictly increasing functions of $i$, that $t_{k}(i)>i$ for all $i \in$ domain $t_{k}$, and that domain $t_{k}=$ domain $f_{k}$ is an initial segment of $E$.

Let $\sigma_{k}$ denote this common domain,

$$
\sigma_{k} \text { is finite and nonvoid. }
$$

Proof. $\sigma_{k} \neq \varnothing$ since $f_{k}(0)$ and $t_{k}(0)$ are defined, so that $0 \in \sigma_{k}$. We will show that if $\sigma_{k}$ is infinite, then $w_{a}^{\prime}$ has an infinite r.e. subset. Now if $\sigma_{k}$ is infinite, then $\sigma_{k}=E$ and $f_{k}(i)$ is defined for all $i \in E$. We claim the following then hold:

(a) $p_{k} f_{k}(i)$ is defined for each $i \in E$,

(b) $p_{k} f_{k}(i) \in w_{a}^{\prime}$ for each $i \in E$. 
To prove (a), assume that $p_{k} f_{k}\left(i^{*}\right)$ is undefined for some $i^{*}$. Then for all $t$, $\pi_{k}^{t} f_{k}\left(i^{*}\right)=0$, so that for $i=i^{*}+1$, Case 1 of the definition of $f_{k}(i)$ fails to occur and $f_{k}(i)$ is undefined, contradicting the assumption that $\sigma_{k}=E$. To prove (b), assume that for some $i^{*}, p_{k} f_{k}\left(i^{*}\right) \in w_{a}$. Then for all sufficiently large $t, \pi_{k}^{t} f_{k}\left(i^{*}\right)>0$ and $p_{k}^{t} f_{k}\left(i^{*}\right) \in w_{a}^{t}$. Let

$$
t^{*}=(\mu t)\left(t>t_{k}\left(i^{*}\right) \& \pi_{k}^{t} f_{k}\left(i^{*}\right)>0 \& p_{k}^{t} f_{k}\left(i^{*}\right) \in w_{a}^{t}\right) .
$$

Now $i^{*}<t_{k}\left(i^{*}\right)<t^{*}$, and $t>t_{k}\left(t^{*}-1\right)>t^{*}-1 \rightarrow t \geqq t^{*}$, so that

$$
(t)\left(t>t_{k}\left(t^{*}-1\right) \rightarrow(E j)_{j<t^{*}}\left(p_{k} f_{k}(j) \in w_{a}^{t}\right)\right) .
$$

Thus for $i=t^{*}$, Case 1 above fails to occur and $f_{k}\left(t^{*}\right)$ is undefined, again contradicting the assumption that $\sigma_{k}=E$.

The set $\left\{p_{k} f_{k}(i) \mid i \in E\right\}$ is evidently r.e.; it is infinite because of (a) and the fact that $p_{k}$ is $1-1$ and $f_{k}$ strictly increasing; and by (b), it is a subset of $w_{a}^{\prime}$. Hence $\sigma_{k}$ being infinite is inconsistent with the hypothesis that $w_{a}^{\prime}$ is immune.

Let $M_{k}$ denote the largest element of $\sigma_{k}$.

$$
(E j)_{j \in \sigma_{k}}\left(f_{k}(j) \notin \text { domain } p_{k} \vee p_{k} f_{k}(j) \in w_{a}\right) \text {. }
$$

Proof. Assume not. Then for all $j \leqq M_{k}, f_{k}(j) \in$ domain $p_{k}$ and $p_{k} f_{k}(j) \notin w_{a}$. Choose $t>t_{k}\left(M_{k}\right)$ so large that $\pi_{k}^{t} f_{k}(j)>0$ for all $j \leqq M_{k}$. Then for this $t$, we have

$$
(j)_{j \leqq M_{k}}\left(\pi_{k}^{t} f_{k}(j)>0 \& p_{k}^{t} f_{k}(j) \notin w_{a}^{t}\right),
$$

so that Case 1 of the definition of $f_{k}(i)$ occurs for $i=M_{k}+1$. Then $M_{k}+1 \in \sigma_{k}$, which is a contradiction.

Let $m_{k}=(\mu j)_{j \in \sigma_{k}}\left(f_{k}(j) \notin\right.$ domain $\left.p_{k} \vee p_{k} f_{k}(j) \in w_{a}\right)$.

$$
(i)_{i \leqq m_{k}}(t)_{t \geqq t_{k}(i)}(j)_{j \leqq i}\left(x(j, k, t)=f_{k}(j)\right)
$$

(i.e., for all sufficiently large $t$,

$$
\left.R_{k}-\gamma^{t-1}=\left\{f_{k}(0), \ldots, f_{k}(i), x(i+1, k, t), x(i+2, k, t), \ldots\right\}\right) .
$$

Proof. By induction on $i$ and $t-t_{k}(i)$ :

$\left(\mathrm{a}_{0}\right)$ For $t=t_{k}(0)$, we have

$$
\begin{aligned}
f_{k}(0) & =(\mu x)\left(x \in R_{k}-\gamma^{u_{k}}\right) \\
& =x\left(0, k, u_{k}+1\right)=x\left(0, k, t_{k}(0)\right) .
\end{aligned}
$$

(bo) Now assume that $f_{k}(0)=x(0, k, t)$ for some $t \geqq t_{k}(0)$. Then

$$
f_{k}(0)=(\mu x)\left(x \in R_{k}-\gamma^{t-1}\right)
$$

and, since $R_{k}-\gamma^{t} \subseteq R_{k}-\gamma^{t-1}$, proving $f_{k}(0)=x(0, k, t+1)$ reduces to showing that $f_{k}(0) \notin \gamma^{t}-\gamma^{t-1}$. Assume otherwise. Then $f_{k}(0) \in \bigcup_{j \leqq t}\{F(j, t), G(j, t), H(j, t)\}$.

Case 1. $(E j)_{j \leqq t}\left(f_{k}(0)=F(j, t) \vee f_{k}(0)=H(j, t)\right)$. Then, since $f_{k}(0) \in R_{k}$ and $t \geqq t_{k}(0)>u_{k}$ we deduce from (2.3.1) and the definition of $u_{k}$ that $f_{k}(0) \in \gamma^{u_{k}} \subseteq \gamma^{t-1}$, which contradicts the induction hypothesis. 
Case 2. Otherwise. Then $(E j)_{j \leqq t}\left(f_{k}(0)=G(j, t)\right)$. Now $0 \in \gamma^{t-1}$ and $f_{k}(0)$ $=x(0, k, t) \in R_{k}-\gamma^{t-1}$ implies $f_{k}(0) \neq 0$, so that by (R5), $f_{k}(0) \in R_{j}$. Since $R_{j} \cap R_{k}=\varnothing$ for $j \neq k$, this implies $j=k$. So $f_{k}(0)=G(k, t)=x(n+1, k, t)$ where $n=n(k, t) \geqq 0$. Then by $(\mathrm{R} 1), f_{k}(0)=x(n+1, k, t)>x(0, k, t)=f_{k}(0)$, which is a contradiction.

Now assume that $0<i \leqq m_{k}$ and that the statement holds for $i-1$, i.e., that $(t)_{t \geqq t_{k}(i-1)}(j)_{j<i}\left(x(j, k, t)=f_{k}(j)\right)$.

$\left(\mathrm{a}_{i}\right)$ For $t=t_{k}(i)$, recall that $x(i, k, t)=(\mu x)\left(x>x(i-1, k, t) \& x \in R_{k}-\gamma^{t-1}\right)$. Since $t_{k}(i)>t_{k}(i-1)$, the induction hypothesis yields $x(i-1, k, t)=f_{k}(i-1)$, so that

$$
x\left(i, k, t_{k}(i)\right)=(\mu x)\left(x>f_{k}(i-1) \& x \in R_{k}-\gamma^{t_{k}^{(i)}-1}\right)=f_{k}(i)
$$

by definition of $f_{k}(i)$.

$\left(b_{i}\right)$ Now assume that $x(i, k, t)=f_{k}(i)$ for some $t \geqq t_{k}(i)$. Then

$$
\begin{aligned}
f_{k}(i) & =(\mu x)\left(x>x(i-1, k, t) \& x \in R_{k}-\gamma^{t-1}\right) \\
& =(\mu x)\left(x>f_{k}(i-1) \& x \in R_{k}-\gamma^{t-1}\right)
\end{aligned}
$$

by the induction hypothesis (on $i)$ and the fact that $t \geqq t_{k}(i)>t_{k}(i-1)$. Then to prove that $f_{k}(i)=x(i, k, t+1)$ it again suffices to show that $f_{k}(i) \notin \gamma^{t}-\gamma^{t-1}$. Assume the contrary; then

$$
f_{k}(i) \in \bigcup_{j \leqq t}\{F(j, t), G(j, t), H(j, t)\}
$$

Case 1. $(E j)_{j \leqq t}\left(f_{k}(i)=F(j, t) \vee f_{k}(i)=H(j, t)\right)$. Then since $t \geqq t_{k}(i)>t_{k}(0)=u_{k}$ we obtain as in $\left(\mathrm{b}_{0}\right)$ above that $f_{k}(i) \in \gamma^{u_{k}} \subseteq \gamma^{t-1}$, which is a contradiction.

Case 2. Otherwise. Then $(E j)_{j \leqq t}\left(f_{k}(i)=G(j, t)\right)$. Again as in $\left(\mathrm{b}_{0}\right)$ above, we conclude that $j=k$ and that $f_{k}(i)=G(k, t)=x(n+1, k, t)$ where $n=n(k, t)$. From $f_{k}(i)=x(i, k, t)$ we then deduce $i=n+1$ or $n(k, t)=i-1$. By (R4) above, $G(k, t)$ $=f_{k}(i) \neq 0$, which, together with the induction hypothesis that

$$
f_{k}(i-1)=x(i-1, k, t)=x(n, k, t),
$$

implies that $\pi_{k}^{t} f_{k}(i-1)=0 \vee p_{k}^{t} f_{k}(i-1) \in w_{a}^{t}$. We show this leads to a contradiction. Now by definition of $t_{k}(i), \pi_{k}^{t_{k}(i)} f_{k}(i-1)>0$ and, since $\pi_{k}^{t}$ is a nondecreasing function of $t, t \geqq t_{k}(i)$ implies $\pi_{k}^{t} f_{k}(i-1)>0$. It follows that $p_{k} f_{k}(i-1)=p_{k}^{t} f_{k}(i-1) \in w_{a}^{t} \subseteq w_{a}$; then by definition of $m_{k}, m_{k} \leqq i-1$, which contradicts the assumption that $0<i \leqq m_{k}$. This completes the induction and the proof of (2.3.4).

(a) $\lim _{t} n(k, t)=m_{k}$,

(b) $R_{k} \cap \gamma^{\prime}=\left\{f_{k}(0), \ldots, f_{k}\left(m_{k}\right)\right\}$.

Proof. (2.3.4) for $i=m_{k}$ yields

$$
(j)_{j \leqq m_{k}}(t)_{t \geqq t_{k}\left(m_{k}\right)}\left(x(j, k, t)=f_{k}(j)\right) .
$$


Recall that $m_{k}=(\mu i)\left(f_{k}(i) \notin\right.$ domain $\left.p_{k} \vee p_{k} f_{k}(i) \in w_{a}\right)$, and choose $v$ so large that

(i) $\pi_{k}^{v} f_{k}(j)>0$ for $0 \leqq j<m_{k}$,

(ii) $v \geqq t_{k}\left(m_{k}\right)>m_{k}$,

(iii) if $f_{k}\left(m_{k}\right) \in$ domain $p_{k}$, then $\pi_{k}^{v} f_{k}\left(m_{k}\right)>0$ and $p_{k}^{v} f_{k}\left(m_{k}\right) \in w_{a}^{v}$.

It is then evident from the definition of $n(k, t)$ that $n(k, t)=m_{k}$ for all $t \geqq v$, which proves (a). To prove (b), note that by (2.3.4), $v \geqq t_{k}\left(m_{k}\right)$ implies

$$
(j)_{j m_{k}}(t)_{t \geqq v}\left(f_{k}(j)=x(j, k, t)\right),
$$

so that $\left\{f_{k}(0), \ldots, f_{k}\left(m_{k}\right)\right\} \subseteq \bigcap_{t \geqq v} R_{k}-\gamma^{t}=\bigcap_{t} R_{k}-\gamma^{t}=R_{k} \cap \gamma^{\prime}$. To prove the converse inclusion, assume $x \in R_{k} \cap \gamma^{\prime}$. Then $x \in R_{k}-\gamma^{v-1}$, which implies $x=x(j, k, v)$ for some $j$. But it is easily seen by induction on $p$ that if

$$
x=x\left(m_{k}+p+1, k, v\right) \quad \text { then } \quad x=G(k, v+p) \in \gamma^{v+p} \subseteq \gamma .
$$

So $x=x(j, k, v) \in R_{k}-\gamma$ only if $j \leqq m_{k}$, in which case $x \in\left\{f_{k}(0), \ldots, f_{k}\left(m_{k}\right)\right\}$.

Proof of Lemma 2.3. Part (a) follows from (2.3.5a). Part (b) follows from (2.3.5b). To prove (c), note that by (2.3.5b), $f_{k}\left(m_{k}\right) \in \gamma^{\prime}$ while by definition of $m_{k}$, either $f_{k}\left(m_{k}\right) \notin$ domain $p_{k}$ or $p_{k} f_{k}\left(m_{k}\right) \in w_{a}$.

LEMMA 2.4. Assume that $0<k$ and that for all $j<k, \lim _{t} n(j, t)=m_{j}$ exists and $c\left(R_{j} \cap \gamma^{\prime}\right)=1+m_{j}$. Then

(a) $\lim _{t} H(k, t)$ exists,

(b) not $w_{a}^{\prime} \prec_{k} \gamma^{\prime}$.

Proof. Let $R=1+\sum_{j<k} 1+m_{j}$ and let $z_{0}, \ldots, z_{R}$ be the least $R+1$ elements of $w_{a}^{\prime}$. Choose $v$ so large that

(i) $(t)_{t \geq v}(j)_{j<k}\left(n(j, t)=m_{j}\right)$,

(ii) $(z)_{z}{z_{R}}_{R}\left(z \in w_{a} \rightarrow z \in w_{a}^{v}\right)$.

Then $(t)_{t>v}(i)_{i \leqq R}\left(r(k, t)=R \quad \& \quad z(i, t)=z_{i}\right)$.

Case 1. $(i)_{i \leqq R}\left(z_{i} \in\right.$ domain $\left.p_{k} \& p_{k}\left(z_{i}\right) \in \bigcup_{j<k} R_{j}\right)$. Let

$$
v^{*}=(\mu t)_{t>v}(i)_{i \leqq R}\left(\pi_{k}^{t}\left(z_{i}\right)>0\right) .
$$

Then for all $t \geqq v^{*}, s(k, t)=R$ and $H(k, t)=0$, which proves (a). To prove (b), note that the hypothesis implies that

$$
c\left(\left(\bigcup_{j<k} R_{j}\right) \cap \gamma^{\prime}\right)=c\left(\bigcup_{j<k}\left(R_{j} \cap \gamma^{\prime}\right)\right)=\sum_{j<k} 1+m_{j}=R .
$$

But $\left\{p_{k}\left(z_{0}\right), \ldots, p_{k}\left(z_{R}\right)\right\}$ is an $(R+1)$-element subset of $\bigcup_{j<k} R_{j}$, which then cannot be contained in $\gamma^{\prime}$, i.e., for some $z_{i} \in w_{a}^{\prime}, p_{k}\left(z_{i}\right) \in \gamma$.

Case 2. Otherwise. Let $i^{*}=(\mu i)_{i \leqq R}\left(z_{i} \notin\right.$ domain $\left.p_{k} \vee p_{k}\left(z_{i}\right) \in \bigcap_{j<k} R_{j}^{\prime}\right)$.

Subcase 2.1. $z_{i^{*}} \notin$ domain $p_{k^{\prime}}$. Let $v^{*}=(\mu t)_{t>v}(i)_{i<i^{*}}\left(\pi_{k}^{t}\left(z_{i}\right)>0\right)$. Then for all $t \geqq v^{*}, s(k, t)=i^{*}$ and $\pi_{k}^{t} z\left(i^{*}, t\right)=0$, so that $H(k, t)=0$, which proves (a). Part (b) holds, since $z_{i *} \in w_{a}^{\prime}$, so that $w_{a}^{\prime} \nsubseteq$ domain $p_{k}$.

Subcase 2.2. Otherwise. Then $z_{i^{*}} \in$ domain $p_{k}$ and $p_{k}\left(z_{i^{*}}\right) \in \bigcap_{j<k} R_{j}^{\prime}$. Let $v^{*}=(\mu t)_{t>v}(i)_{i \leqq i} \cdot\left(\pi_{k}^{t}\left(z_{i}\right)>0\right)$. Then for all $t \geqq v^{*}, s(k, t)=i^{*}$ and $H(k, t)=p_{k}\left(z_{i}\right)$, which proves (a). Part (b) follows since $z_{i^{*}} \in w_{a}^{\prime}$ while $p_{k}\left(z_{i^{*}}\right)=H\left(k, v^{*}\right) \in \gamma^{v^{*}} \subseteq \gamma$. 
LEMMA 2.5. For each $k$, the following hold:
$\left(\mathrm{A}_{k}\right)$ not $w_{a}^{\prime} \prec_{k} \gamma^{\prime}$,
$\left(\mathrm{B}_{k}\right)$ not $\gamma^{\prime} \prec_{k} w_{a}^{\prime}$,
$\left(\mathrm{C}_{k}\right) \lim _{t} H(k, t)$ exists,
$\left(\mathrm{D}_{k}\right) \lim _{t} n(k, t)$ exists,
$\left(\mathrm{E}_{k}\right) c\left(R_{k} \cap \gamma^{\prime}\right)=1+\lim _{t} n(k, t)$.

Proof. By induction on $k$. Lemma 2.2 gives $\left(\mathrm{A}_{0}\right)$ and $\left(\mathrm{C}_{0}\right)$. Lemma 2.3 then gives $\left(D_{0}\right),\left(E_{0}\right)$, and $\left(B_{0}\right)$, which completes the base step. Now assume $0<k$ and that $\left(\mathrm{A}_{j}\right),\left(\mathrm{B}_{j}\right),\left(\mathrm{C}_{j}\right),\left(\mathrm{D}_{j}\right)$, and $\left(\mathrm{E}_{j}\right)$ hold for all $j<k$. Then $\left(\mathrm{C}_{k}\right)$ and $\left(\mathrm{A}_{k}\right)$ follow by Lemma 2.4, which by Lemma 2.3 implies $\left(D_{k}\right),\left(E_{k}\right)$, and $\left(B_{k}\right)$. The conclusions thus hold for all $k$.

LEMMA 2.6. $\gamma^{\prime}$ has no infinite r.e. subset.

Proof. Let $w_{k}$ be an infinite r.e. set.

Case 1. $w_{k} \subseteq \bigcup_{j<k} R_{j}$. Then $w_{k} \cap \gamma^{\prime} \subseteq \bigcup_{j \leqq k}\left(R_{j} \cap \gamma^{\prime}\right)$ which by parts (D) and (E) of Lemma 2.5 is a finite union of finite sets. So $w_{k} \cap \gamma^{\prime}$ is finite, which implies $w_{k} \cap \gamma \neq \varnothing$.

Case 2. Otherwise. Then $w_{k}-\bigcup_{j \leqq k} R_{j} \neq \varnothing$, which by Lemma 2.1(b) implies $w_{k} \cap \gamma \neq \varnothing$.

End of proof of Theorem 2. Let $X=\left\langle w_{a}^{\prime}\right\rangle \in \Lambda_{z}-E$. The set $\gamma$ constructed above is evidently r.e., since effective instructions were given for generating it. Let $Y=\left\langle\gamma^{\prime}\right\rangle$; then by Lemma 2.5(A) and (B), $Y \$ X$ and $X \$ Y$. By Lemma 1.1, this implies $Y \notin E$, so that $\gamma^{\prime}$ is infinite. Then by Lemma 2.6, $\gamma^{\prime}$ is immune and $Y \in \Lambda_{z}$. This completes the proof of the theorem.

Note that the instructions for generating $\gamma$ are uniform in $a$, so that an index of $Y$ can be effectively computed given one of $X$; i.e., there is a recursive function $h$ such that if $X=\left\langle w_{a}^{\prime}\right\rangle \in \Lambda_{z}-E$ and $Y=\left\langle w_{h(a)}^{\prime}\right\rangle$, then $Y \in \Lambda_{z}$ and $Y$ is incomparable to $X$. In [3], a sequence $X_{1}, X_{2}, \ldots$ of elements of $\Lambda_{z}$ is called r.e. if there is a recursive function $f$ such that $X_{i}=\left\langle w_{f(i)}^{\prime}\right\rangle$ for each $i \in E$, and a Gödel number of $f$ is called an index of $\left\{X_{i}\right\}$. A set $P$ of elements of $\Lambda_{z}$ is called productive if there is a recursive function $f$ such that if $\left\{X_{i}\right\}$ is an r.e. sequence of elements of $P$ with index $e$, then $\left\langle w_{f(e)}^{\prime}\right\rangle \in P-\left\{X_{i}\right\}$. Using standard methods of "interweaving priorities," the techniques of Theorem 2 can be modified so as to effectively produce, given an r.e. sequence $\left\{X_{i}\right\} \subseteq \Lambda_{z}-E$, an element $Y \in \Lambda_{z}$ which is incomparable to $X_{i}$ for each $i$. This leads to a proof of

THEOREM 3. Every maximal set of mutually incomparable elements of $\Lambda_{z}-E$ is productive.

\section{REFERENCES}

1. J. C. E. Dekker and J. Myhill, The divisibility of isols by powers of primes, Math. Z. 73 (1960), 127-133.

2. - Recursive equivalence types, Univ. of California Publications in Mathematics (New Series) 3, No. 3 (1960), 67-214. 
3. L. Hay, The co-simple isols, Ann. of Math. 83 (1966), 231-256.

4. S. C. Kleene, Introduction to metamathematics, Van Nostrand, New York, 1952.

5. A. Nerode, Diophantine correct nonstandard models in the isols, Ann. of Math. 84 (1966), $421-432$.

6. - Extensions to isols, Ann. of Math. 73 (1961), 362-403.

7. H. Rogers, Jr., Computing degrees of unsolvability, Math. Ann. 138 (1959), 125-140.

8. G. E. Sacks, Degrees of unsolvability, Annals of Mathematics Studies No. 55, Princeton Univ. Press, Princeton, N. J., 1963.

Mount Holyoke College,

South Hadley, MasSaChusetTs 